\title{
A NEW CHARACTERIZATION OF THE BRUHAT DECOMPOSITION
}

\author{
YOSHIFUMI KATO
}

\section{§. Introduction}

By an algebraic homogeneous space, we mean the factor space $X=$ $G / P$, where $G$ is a simply-connected, complex, semi-simple Lie group and $P$ is a parabolic subgroup of $G$. Many typical manifolds such as the projective spaces and the Grassmann varieties belong to this class of manifolds. For instance, the Grassmann variety $G(k, n)$ can be expressed as $S L(n+1, C) / P$, where $P$ is a maximal parabolic subgroup of $S L(n+1, C)$ leaving a suitable $k+1$ dimensional subspace invariant. In this paper, we devote ourselves to study the Bruhat decomposition of an algebraic homogeneous space $X=G / P$. For that purpose, in the first place, we construct a holomorphic vector field $V_{H}$ on $X$ for an element $H$ of the positive Weyl chamber as follows

$$
\left(V_{H} f\right)(\bar{g})=\lim _{s \rightarrow 0} \frac{f(\exp (\varepsilon H) \bar{g})-f(\bar{g})}{\varepsilon},
$$

where $\bar{g} \in X$ and $f$ is a local function around $\bar{g}$. Then the vector field $V_{H}$ vanishes only at the isolated points and in fact at the quotient set $W^{1}=$ $W / W_{1}$, which is naturally embedded into $X=G / P$ as the set of all fixed points of the left $T$ action. Here $W$ and $W_{1}$ are the Weyl groups associated to $G$ and $P$ respectively. And there is a nice coordinate neighborhood ( $\left.\mathfrak{w} \bar{N}^{*}, \phi^{-1} \circ \psi^{-1} \circ \mathfrak{w}^{-1}\right)$ around each $\mathfrak{w} \in W^{1}$ such that;

1) The set $\mathfrak{w} \bar{N}^{*}$ is a $T$ invariant Zariski open set. Let $\mathfrak{w}_{0}$ be the element of $W$ whose length $\ell\left(\mathfrak{w}_{0}\right)$ is maximal among all. Then the set $\mathfrak{w}_{0} \bar{N}^{*}$ coincides with the cell of maximal dimension appeared in the Bruhat decomposition of $X$.

2) The set $\mathfrak{w} \bar{N}^{*}$ is mapped by $\phi^{-1} \circ \psi^{-1} \circ \mathfrak{w}^{-1}$ onto the Lie algebra $\mathfrak{n}^{*}$ of the Lie group $N^{*}$ and hence we can write as

Received November 26, 1979. 


$$
\phi^{-1} \circ \psi^{-1} \circ \mathfrak{W}^{-1}\left(\mathfrak{W} \bar{n}^{*}\right)=\sum_{\alpha \in \Delta\left(\mathfrak{n}^{*}\right)} z_{\alpha}\left(\mathfrak{W} \bar{n}^{*}\right) X_{\alpha} .
$$

3) With respect to the local coordinate $\left\{z_{\alpha}\left(\mathfrak{w} \bar{n}^{*}\right)\right\}_{\alpha \in \Delta\left(n^{*}\right)}$, the vector field $V_{H}$ can be written explicitly as follows

$$
V_{H}=\sum_{\alpha \in \Delta\left(\mathrm{n}^{*}\right)}(\mathfrak{w} \alpha)(H) z_{\alpha} \frac{\partial}{\partial z_{\alpha}} .
$$

From the above expression of $V_{H}$ on $\mathfrak{w} \bar{N}^{*}$, any flow $P(t)$, parametrized by $t \in C$, of $V_{H}$ on $\mathfrak{w} \bar{N}^{*}$ can be written as $z_{\alpha}(P(t))=c_{\alpha} e^{(w \alpha)(H) t}, c_{\alpha} \in C$, for $\alpha \epsilon$ $\Delta\left(\mathfrak{n}^{*}\right)$.

Every algebraic homogeneous space $X$ has the distinguished cellular decomposition $X=\bigcup_{\mathfrak{w} \in W^{1}} X_{\mathfrak{w}}$ parametrized by $W^{1}$, which is called the Bruhat one of $X$. We recall the definition precisely in section 2 . The new point of view in this note is to understand that any Bruhat cell $X_{\mathfrak{w}}, \mathfrak{w} \in W^{1}$, consists of some flows $P(t)$ of $V_{H}$. We embed the space $X$ into a certain projective space $\boldsymbol{P}^{N}$ and construct a vector field $V_{H}^{A}$ on $\boldsymbol{P}^{N}$ whose restriction to $X$ coincides with the vector field $V_{H}$. And we indentify the flow $P(t)$ as that of $V_{H}^{A}$ lying entirely on $X$ and investigate the behavior of it. As a result we obtain a rule to seek for the limit points $\lim _{t \rightarrow \pm \infty} P(t)$. And we show that to compactify $X_{\mathfrak{w}}$ to $\bar{X}_{\mathfrak{w}}$, it is enough to attach such limit points $\lim _{t \rightarrow \pm \infty} P(t)$ successively. The set $\bar{X}_{\mathfrak{w}}$ is a subvariety of $X$ and is called the generalized Schubert variety. The family of subvarieties $\bar{X}_{w}$, $\mathfrak{w} \in W^{1}$, has a lot of important meanings for the geometry of $X=G / P$. For instance, the homology classes of $\left\{X_{\mathfrak{w}}\right\} \mathfrak{w} \in W^{1}$ form the free $Z$-basis of the integral homology group $H_{*}(X, Z)$ of $X$. In case $X=\operatorname{Gr}(k, n)$, the classical Schubert calculus is to study the ring structure of $H_{*}(X, Z)$ by using the basis.

Further we give the following results in Theorem 4.9 and Theorem 4.12.

1) We give a new geometrical meaning to the reflections $S_{\alpha}, \alpha \in \Delta$.

2) We obtain a simple necessary sufficient condition to determine the Bruhat ordering $\leq$ on $W^{1}$.

3) From 1) and 2), we clarify what Bruhat cells of lower dimension should be attached to the boundary of $X_{\mathfrak{w}}$ of the appointed direction to compactify $X_{w}$.

It seems interesting to study the singularities of $\bar{X}_{\mathfrak{w}}$ as a sequel of this note. And we also wish to clarify the relations between our results and the representation theory of nilpotent Lie algebras.

This paper is divided into four parts. 
1. Semisimple Lie algebras.

2. Bruhat decomposition of $G / P$.

3. Vector field $V_{H}$.

4. Proof of our theorems.

The author wishes to express his thanks to Profs. H. Morikawa, K. Aomoto and $\mathrm{H}$. Umemura for their valuable suggestions.

\section{§1. Semisimple Lie algebras}

First we fix the terminology and recall the fundamental facts of semisimple Lie algebras.

Let $g$ be a complex semisimple Lie algebra. We choose a real Lie subalgebra $t$ of $g$ which satisfies;

1) $g=t+i$ is a direct sum,

2) the Killing form ( , ) is negative definite on $t$.

We fix the above chosen algebra $t$ later and call it a compact form of $g$. Let $q=i$. Then $q$ is a real subspace of $\mathfrak{g}$ and the Killing form (, ) is positive definite there. We introduce a $*$-operation, which is conjugate linear, as follows

$$
(u+i v)^{*}=u-i v, \quad u, v \in \mathfrak{q} .
$$

From the definition, $*^{2}$ is the identity operation of $g$. We denote

$$
\{X, Y\}=\left(X, Y^{*}\right), \quad X, Y \in \mathfrak{g},
$$

then the form $\{$,$\} becomes a positive definite hermitian form on \mathfrak{g}$. For any vector subspace $\mathfrak{a}$ of $\mathfrak{g}$, we set

$$
\begin{aligned}
\mathfrak{a}^{0} & =\{X \in \mathfrak{g} \mid(X, Y)=0 \text { for any } Y \in \mathfrak{a}\} \\
& =\left\{X \in \mathfrak{g} \mid\left\{X, Y^{*}\right\}=0 \text { for any } Y \in \mathfrak{a}\right\} .
\end{aligned}
$$

Let $\mathfrak{b}$ be a Borel subalgebra of $\mathfrak{g}$ and fix it once and for all. Put $\mathfrak{h}$ $=\mathfrak{b} \cap \mathfrak{b}^{*}$. Then $\mathfrak{h}$ is a Cartan subalgebra of $\mathfrak{g}$. The rank $l$ of $\mathfrak{g}$ is, by definition, the dimension of $\mathfrak{h}$. Let $\mathfrak{h}_{R}$ be the real part of $\mathfrak{h}$ with respect to the *-operation. We denote by $\hat{\mathfrak{h}}_{R}$ the dual vector space of $\mathfrak{G}_{R}$. The root system $\Delta$ of $\mathfrak{h}$ in $\mathfrak{g}$ is contained in $\hat{\mathfrak{h}}_{R}$. The set $\Delta$ is divided into two classes, positive roots $\Delta_{+}$and negative roots $\Delta_{-}$with respect to $\mathfrak{b}$;

1) $\Delta=\Delta_{+} \cup \Delta_{-}$, disjoint union,

2) $\Delta_{-}=-\Delta_{+}$.

3) if $\alpha, \beta \in \Delta_{+}$(respectively $\Delta_{-}$) and $\alpha+\beta \in \Delta$ then $\alpha+\beta \in \Delta_{+}\left(\Delta_{-}\right)$.

Each connected component of the set 


$$
\left\{H \in \mathfrak{h}_{R} \mid \alpha(H) \neq 0 \text { for } \alpha \in \Delta_{+}\right\}
$$

is called a Weyl chamber. The following set

$$
\mathfrak{h}_{\boldsymbol{R}}^{+}=\left\{H \in \mathfrak{h}_{\boldsymbol{R}} \mid \alpha(H)>0 \text { for } \alpha \in \Delta_{+}\right\}
$$

is, by definition, the positive Weyl chamber. Then conversely for any $H \in \mathfrak{h}_{\boldsymbol{R}}^{+}$, we have

$$
\Delta_{+}=\{\alpha \in \Delta \mid \alpha(H)>0\} .
$$

We denote by $\Pi=\left\{\alpha_{1}, \cdots, \alpha_{l}\right\}$ the set of all simple roots with respect to $\Delta_{+}$and take the set as the basis of $\hat{\mathfrak{h}}_{\boldsymbol{R}}$. We can write any root $\phi \in \Delta$ as $\phi=\sum n_{\alpha_{i}}(\phi) \alpha_{i}$ where $n_{\alpha_{i}}(\phi)$ are non negative or non positive integers according to $\phi \in \Delta_{+}$or $\Delta_{-}$.

We decompose the algebra $g$ into root spaces;

$$
\mathfrak{g}=\mathfrak{h}+\sum_{\alpha \in \Delta_{+}} \mathfrak{g}_{\alpha}+\sum_{\beta \in \Delta_{-}} \mathfrak{g}_{\beta}
$$

where

$$
\mathfrak{b}=\mathfrak{h}+\sum_{\alpha \in \Delta_{+}} \mathfrak{g}_{\alpha}
$$

For any $\alpha \in \Delta, \operatorname{dim} \mathfrak{g}_{\alpha}=1$ and from the definition of $\mathfrak{g}_{\alpha}$, it follows

$$
\operatorname{ad}(H)(X)=[H, X]=\alpha(H) X, \quad X \in \mathfrak{g}_{\alpha}, \quad H \in \mathfrak{h} .
$$

We can choose the elements $H_{\alpha} \in \mathfrak{h}, X_{\alpha} \in \mathfrak{g}_{\alpha}, X_{-\alpha} \in \mathfrak{g}_{-\alpha}$ for $\alpha \in \Delta_{+}$in the way

$$
\begin{aligned}
& \left(H, H_{\alpha}\right)=\alpha(H), \quad H \in \mathfrak{h}, \\
& \left(X_{\alpha}, X_{\beta}\right)= \begin{cases}1 & \text { if } \alpha=-\beta \\
0 & \text { if } \alpha \neq-\beta,\end{cases}
\end{aligned}
$$

and consequently

$$
\left[X_{\alpha}, X_{-\alpha}\right]=H_{\alpha} \text {. }
$$

Since the elements $H_{\alpha}, \alpha \in \Delta_{+}$, are uniquely determined if we put

$$
(\alpha, \beta)=\left(H_{\alpha}, H_{\beta}\right), \quad \alpha, \beta \in \Delta_{+},
$$

we can define a positive definite symmetric bilinear form $($,$) on \hat{\mathfrak{h}}_{\boldsymbol{R}}$.

Let $[\mathfrak{P}]$ denote the set of all parabolic subalgebras which contain $\mathfrak{b}$. For each $\mathfrak{p} \in[\mathfrak{P}]$, we know the following decomposition of $\mathfrak{g}$. See [6].

Theorem 1.1. Let $\mathfrak{p} \in[\mathfrak{P}]$. We put $\mathfrak{n}=\mathfrak{p}^{0}$. Then $\mathfrak{n}$ is the maximal 
nilpotent ideal of $\mathfrak{p}$ and also the set of all nilpotent elements in the radical of $\mathfrak{p}$. If we put $\mathfrak{g}_{1}=\mathfrak{p} \cap \mathfrak{p}^{*}$ then $\mathfrak{g}$ is decomposed into;

$$
\mathfrak{g}=\mathfrak{n}^{*}+\mathfrak{g}_{1}+\mathfrak{n}
$$

where

$$
\mathfrak{p}=\mathfrak{g}_{1}+\mathfrak{n} .
$$

Moreover $\mathfrak{g}_{1}$ lies in the normalizers of both $\mathfrak{n}$ and $\mathfrak{n}^{*}$, i.e., $\left[\mathfrak{g}_{1}, \mathfrak{n}\right] \subset \mathfrak{n}$ and $\left[\mathfrak{g}_{1}, \mathfrak{n}^{*}\right] \subset \mathfrak{n}^{*}$.

For any vector space $\mathfrak{a}$ which is invariant by the adjoint representation of $\mathfrak{h}$, we define the subset $\Delta(\mathfrak{a}) \subset \Delta$ as follows

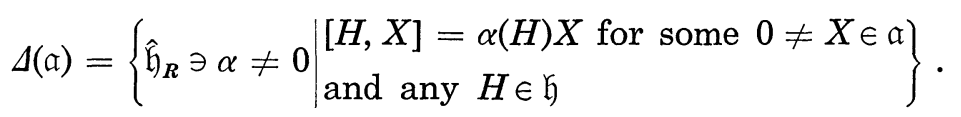

Since we have $\mathfrak{p} \supset \mathfrak{b} \supset \mathfrak{h}$, we can see $\mathfrak{p}$ as soon as we know $\Delta(\mathfrak{p})$ or in fact $\Delta(\mathfrak{p}) \cap \Delta_{-}$. The following theorem is also in [6].

THEOREM 1.2. 1) There exists a one-to-one mapping, $\mathfrak{p} \rightarrow \Pi(\mathfrak{p})$, from [P] onto the set of all subset of $\Pi$.

2) The subset $\Pi(\mathfrak{p})$ of $\Pi$ satisfies

$$
\Delta(\mathfrak{p}) \cap \Delta_{-}=\left\{\phi \in \Delta_{-} \mid n_{\alpha_{i}}(\phi)=0 \text { for all } \alpha_{i} \in \Pi(\mathfrak{p})\right\} .
$$

Since there are $l$ elements in $\Pi$, the number of [ß] is exactly $2^{l}$. The Lie algebras $\mathfrak{g}_{1}, \mathfrak{n}$ and $\mathfrak{n}^{*}$ are all invariant by the adjoint action of $\mathfrak{h}$. And we have;

$$
\begin{aligned}
& \Delta\left(\mathfrak{g}_{1}\right)=\left\{\phi \in \Delta \mid n_{\alpha_{i}}(\phi)=0 \text { for } \alpha_{i} \in \Pi(\mathfrak{p})\right\}, \\
& \Delta(\mathfrak{n})=\left\{\phi \in \Delta_{+} \mid n_{\alpha_{i}}(\phi)>0 \text { for } \alpha_{i} \in \Pi(\mathfrak{p})\right\}, \\
& \Delta\left(\mathfrak{n}^{*}\right)=-\Delta(\mathfrak{n}) .
\end{aligned}
$$

\section{§ 2. Bruhat decomposition of $G / P$}

We use the notations in $\S 1$., i.e., $g$ denotes a semisimple Lie algebra, $\mathfrak{b}$ a Borel subalgebra, $\mathfrak{h} \subset \mathfrak{b}$ a Cartan subalgebra, $\cdots$, etc.

Let $G$ be a simply-connected Lie group whose Lie algebra is $\mathrm{g}$. Let $B$ be the Borel subgroup of $G$ whose Lie algebra is $\mathfrak{b}$. Let $T$ be the maximal torus with Lie algebra $\mathfrak{h}$. We denote by $P$ the parabolic subgroup corresponding to $\mathfrak{p} \in[\mathfrak{P}]$. Then we have $G \supset P \supset B \supset T$. We call the factor space $X=G / P$ an algebraic homogeneous space. The space $X$ 
is a compact kähler manifold and in fact is a projective algebraic manifold. We recall how to embed the space $X=G / P$ into a projective space in section 4.

Let $G_{1}, N$ and $N^{*}$ be the Lie subgroups of $G$ whose Lie algebras are $\mathfrak{g}_{1}, \mathfrak{n}$ and $\mathfrak{n}^{*}$ respectively. Then the group $P$ is the semidirect product of $G_{1}$ and $N$, i.e.;

$$
\begin{aligned}
& P=G_{1} \cdot N, \\
& G_{1} \cap N=\{I\}, \\
& N \text { is normal in } P .
\end{aligned}
$$

And the groups $P$ and $N^{*}$ satisfy

$$
P \cap N^{*}=\{I\}
$$

See [11].

We denote by $N(T)$ the normalizer of $T$ in $G$. We call the group $W=N(T) / T$ the Weyl group of $G$ with respect to $T$. We define a subgroup $W_{1}$ of $W$ and the quotient $W^{1}$ in the following

$$
\begin{aligned}
& W_{1}=N(T) \cap P / T, \\
& W^{1}=W / W_{1}=N(T) / N(T) \cap P .
\end{aligned}
$$

The group $N(T)$ acts on $T, \mathfrak{h}$ and $\Delta$ and the formulae

$$
\mathfrak{w} \cdot \exp H \cdot \mathfrak{w}^{-1}=\exp (\operatorname{Ad}(\mathfrak{w}) H), \quad\left(\operatorname{Ad}(\mathfrak{w})^{*} \alpha\right)(H)=\alpha\left(\operatorname{Ad}(\mathfrak{w})^{-1}(H)\right),
$$

for $\mathfrak{w} \in N(T), H \in \mathfrak{h}, \alpha \in \Delta$, are valid. But the actions of $\mathfrak{w} \in T$ are all trivial so we can regard as the group $W=N(T) / T$ acts on $T, \mathfrak{h}$ and $\Delta$. For simplicity, we use the same letter $\mathfrak{w}$ for $\mathfrak{w}, \mathrm{Ad}(\mathfrak{w})$ and $\mathrm{Ad}(\mathfrak{w})^{*}$. The action of $W$ on $\Delta$ can be naturally extended on $\hat{\mathfrak{h}}_{\boldsymbol{R}}$ and then any element of $W$ acts as an orthogonal transformation with respect to the norm (, ) introduced in section 1 . For any $\alpha \in \Delta$, we define the reflection $S_{\alpha}$;

$$
S_{\alpha}(\phi)=\phi-\frac{2(\alpha, \phi)}{(\alpha, \alpha)} \alpha, \quad \phi \in \hat{\mathfrak{h}}_{R},
$$

then $S_{\alpha}$ belongs to $W$. Further the Weyl group $W$ is generated by the reflections $S_{\alpha_{i}}, \alpha_{i} \in \Pi$, and the subgroup $W_{1}$ is generated by the reflections $S_{\alpha j}, \alpha_{j} \in \Pi-\Pi(\mathfrak{p})$. The length $\ell(\mathfrak{w})$ of $\mathfrak{w} \in W$ is the number of reflections appeared in the reduced expressions of $\mathfrak{w}$ by using the reflections $S_{\alpha_{i}}, \alpha_{i}$ $\in \Pi$. 
Remark 2.1. By virtue of (1.8), the group $W_{1}$ keeps the set $\Delta\left(n^{*}\right)$ invariant.

Remark 2.2. We fix some representatives $\left\{\mathfrak{w}_{1}, \cdots, \mathfrak{w}_{k}\right\}$ of $W^{1}$ in $N(T)$. Here $k={ }^{\sharp} W^{1}$. And we sometimes equate the representatives with those of $W^{1}=W / W_{1}$ in $W$ and further with the set $W^{1}$ itself.

Every semisimple Lie group $G$ admits the following double coset decomposition and there are three ways to describe;

$$
G=\bigcup_{\mathfrak{w} \in W^{1}} N \mathfrak{r} P=\bigcup_{\mathfrak{w} \in W^{1}} M \mathfrak{m} P=\bigcup_{\mathfrak{w} \in W^{1}} P \mathfrak{w} P,
$$

where $M$ is the maximal nilpotent Lie group of $B$ and $\mathfrak{w}$ runs over all the above chosen representatives. Let $X_{\mathfrak{w}}=N \mathfrak{w} P / P$ be the image of $N \mathfrak{w} P$ in $X$. Then $X_{\mathfrak{w}}$ is a cell and the dimension of $X_{\mathfrak{w}}$ is represented in terms of the length $\ell(\mathfrak{w})$ of $\mathfrak{w}$. Confer [1] or Proposition 4.1 in this paper. From (2.6) $X_{\mathfrak{w}}$ can be considered as the $N, M$ or $P$ orbit of $\overline{\mathfrak{w}}$ in $X$. Then the space $X$ is decomposed into;

$$
X=\bigcup_{w \in W^{1}} X_{\mathfrak{w}}
$$

and we call this decomposition the Bruhat one of $X$. The topological closure $\bar{X}_{\mathfrak{w}}$ of $X_{\mathrm{w}}$, that is also the Zariski closure, is a subvariety of $X$ and is called a generalized Schubert variety. As a fundamental character, the homology classes of $\left\{\bar{X}_{\mathrm{w}}\right\}_{\mathrm{w} \in W^{1}}$ form the free $Z$-basis of the integral homology group $H_{*}(X, Z)$ of $X$.

In the set $W^{1}$, we can introduce a partial ordering $\leq$, called the Bruhat ordering;

$$
\mathfrak{w}_{1} \leq \mathfrak{w}_{2} \text { if and only if } \bar{X}_{\mathfrak{w}_{1}} \subset \bar{X}_{\mathfrak{w}_{2}}
$$

for $\mathfrak{w}_{1}, \mathfrak{w}_{2} \in W^{1}$. By using this ordering, we can write;

$$
\bar{X}_{\mathfrak{w}}=\bigcup_{w^{\prime} \leq w} X_{w^{\prime}} \cdot
$$

Namely, to take the closure $\bar{X}_{\mathrm{w}}$ of $X_{\mathrm{w}}$, it suffices that we attach suitable Bruhat cells of lower dimension to the boundary of $X_{\mathfrak{w}}$ successively. Though this ordering is relatively easily defined, it is not easy to judge the order of arbitrarily given two elements $\mathfrak{w}_{1}, \mathfrak{w}_{2} \in W^{1}$. Hence we wish to restate the ordering in another easily understandable words. And further we wish to make the geometrical meanings of the ordering clear. Roughly speaking, for that purpose there exist two ways until now, firstly 
the way to compare the reduced expressions of $\mathfrak{w} \in W^{1}$ by using the reflections $S_{\alpha_{i}}, \alpha_{i} \in \Pi$, and secondly the way to compare the inclusion relation among Verma modules $M(\mathfrak{w} \Lambda)$ associated to $\mathfrak{w} \in W^{1}$. Here $\Lambda$ is the highest weight which determines the embedding of $X$ into the projective space $\boldsymbol{P}^{N}(\boldsymbol{V})$. See [3].

But as will be shown in section 4 , we take a different approach. We first show that any Bruhat cell can be written as the union of suitable flows $P(t)$ of the vector field $V_{H}$ on $X$. The vector field $V_{H}$ is defined in the next section. We investigate the behavior of the flows and show what Bruhat cells of lower dimension should be attached to the boundary of the Bruhat cell $X_{w}$ of the appointed direction for the compactification of $X_{w}$. We give a simple necessary-sufficient condition to determine the ordering $\leq$ in Theorem 4.12 and ties the two ways stated above in some sence.

\section{§3. Vector field $V_{H}$}

We first prove the following proposition.

Proposition 3.1. Let us act the maximal torus $T$ on $X=G / P$. Then the quotient set $W^{1}=W / W_{1}=N(T) / N(T) \cap P$ is naturally realized as the set of all $T$ fixed points in $X$.

Proof. An element $\bar{g} \in X$ is fixed by the action of $T$ if and only if $g^{-1} T g \subset P$ where $g$ is a representative of $\bar{g}$ in $G$. The group $g^{-1} T g$ is also a maximal torus of $G$ contained in $P$ and hence we have

$$
g^{-1} T g=p T p^{-1}
$$

for some $p \in P$. See [9]. This means $g p \in N(T)$. Hence $\bar{g}$ defines a coset $\widetilde{g p}$ in $W^{1}$. If $\bar{g}$ and $\bar{g}^{\prime}$ define the same coset in $W^{1}$, we can write $g p=$ $g^{\prime} p^{\prime} p^{\prime \prime}$ for some $p, p^{\prime} \in P$ and $p^{\prime \prime} \in N(T) \cap P$. So we have $\bar{g}=\bar{g}^{\prime}$ in $X$. If we take any element $\mathfrak{w} \in N(T)$, the coset corresponding to $\overline{\mathfrak{w}}$ is $\tilde{\mathfrak{w}} \in W^{1}$. So the mapping is onto. The proof is completed.

Let us consider the following diagram

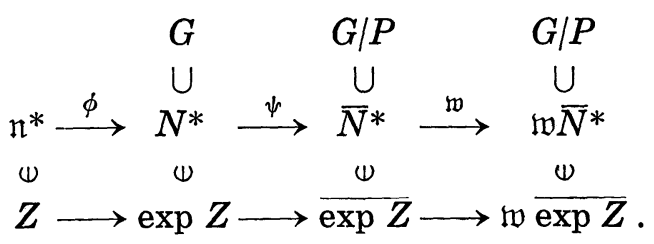


We take the elements $X_{\alpha}, \alpha \in \Delta\left(\mathfrak{n}^{*}\right)$, chosen in section 1 , as the basis of $\mathfrak{n}^{*}$ and write an element $Z$ of $\mathfrak{n}^{*}$ as $Z=\sum_{\alpha \in \Delta\left(\mathfrak{n}^{*}\right)} z_{\alpha} X_{\alpha}$. Since the Lie algebra $\mathfrak{n}^{*}$ is nilpotent, we have $\log (\exp Z)=Z$ and hence the mapping $\phi$ is one-to-one and onto. Since $N^{*} \cap P=\{I\}$, the mapping $\psi$ is also one-to-one. See (2.4). The left multiplication by $\mathfrak{t}$ is clearly one-to-one. Hence we can take the pair $\left(\mathfrak{m} \bar{N}^{*}, \phi^{-1} \circ \psi^{-1} \circ \mathfrak{w}^{-1}\right)$ as a coordinate neighborhood around $\mathfrak{w} \in W^{1}$ and then $\left\{z_{\alpha}\left(\mathfrak{w} \bar{n}^{*}\right)\right\}_{\alpha \in \Delta\left(\mathrm{n}^{*}\right)}$ becomes the local coordinate.

Remark 3.2. Let $\mathfrak{w}_{0}$ be the element of $W$ whose length $\ell\left(\mathfrak{w}_{0}\right)$ is maximal among all. Then since $\mathfrak{w}_{0}^{-1} N \mathfrak{w}_{0}=N^{*}, \mathfrak{w}_{0} \bar{N}^{*}=N \mathfrak{w}_{0} P / P=X_{\mathfrak{w}_{0}}$. So the set $\mathfrak{w}_{0} \bar{N}^{*}$ is the Bruhat cell of maximal dimension and is a Zariski open set.

We sum up our assertions.

TheOREm 3.3. The quotient set $W^{1}=W / W_{1}$ can be naturally embedded into $X=G / P$ as the set of all T-fixed points and the pair ( $10 \bar{N}^{*}, \phi^{-1} \circ \psi^{-1}$ 。 $\left.\mathfrak{w}^{-1}\right)$ is a coordinate neighborhood around $\mathfrak{w} \in W^{1}$. The sets $\mathfrak{w} \bar{N}^{*}, \mathfrak{w} \in W^{1}$, are all $T$ invariant Zariski open sets. In fact if we multiply $\exp H \in T$ from the left side on $\mathfrak{w} \bar{N}^{*}$, the local coordinate $\left\{z_{\alpha}\left(\mathfrak{w} \bar{n}^{*}\right)\right\}_{\alpha \in \Delta\left(\mathfrak{n}^{*}\right)}$ changes to $\left\{e^{(w \alpha)(H)}\right.$ $\left.\cdot z_{\alpha}\left(\mathfrak{n} \bar{n}^{*}\right)\right\}_{\alpha \in \Delta\left(\mathfrak{n}^{*}\right)}$. Further the space $X$ is completely covered with the family of the open sets $\left\{\mathfrak{w} \bar{N}^{*}\right\}_{\mathfrak{w} \in W^{1}}$, i.e., $X=\bigcup_{\mathfrak{w} \in W^{1}} \mathfrak{w} \bar{N}^{*}$.

Proof. The first and second sentences have been proved. Since for $\exp Z \in N^{*}$ we have

$$
\begin{aligned}
\exp H \cdot \mathfrak{w} \exp Z \cdot P & =\mathfrak{w}^{-1} \exp H \cdot \mathfrak{w} \exp Z \cdot \mathfrak{w}^{-1} \exp (-H) \mathfrak{w} \cdot P \\
& =\mathfrak{w} \exp \left(\mathfrak{w}^{-1}(H)\right) \exp Z \exp \left(-\mathfrak{w}^{-1}(H)\right) \cdot P \\
& =\mathfrak{w} \exp \left(\operatorname{Ad}\left(\exp \left(\mathfrak{w}^{-1}(H)\right)\right) Z\right) \cdot P \\
& =\mathfrak{w} \exp \left(\operatorname{Exp}\left(\operatorname{ad}\left(\mathfrak{w}^{-1}(H)\right)\right) Z\right) \cdot P
\end{aligned}
$$

and

$$
\operatorname{Exp}\left(\operatorname{ad}\left(\mathfrak{w}^{-1}(H)\right)\right) \cdot Z \in \mathfrak{n}^{*}
$$

so

$$
\begin{aligned}
\left(\phi^{-1} \circ \psi^{-1} \circ \mathfrak{w}^{-1}\right)(\exp H \cdot \mathfrak{w} \overline{\exp Z}) & =\log \left(\mathfrak{w}^{-1} \mathfrak{w} \exp \left(\operatorname{Exp}\left(\operatorname{ad}\left(\mathfrak{w}^{-1}(H)\right)\right) \cdot Z\right)\right) \\
& =\operatorname{Exp}\left(\operatorname{ad}\left(\mathfrak{w}^{-1}(H)\right)\right) \cdot Z .
\end{aligned}
$$

If we write $Z=\sum_{\alpha \in \Delta\left(\mathfrak{n}^{*}\right)} z_{\alpha} X_{\alpha}$, we have 


$$
\begin{aligned}
\operatorname{ad}\left(\mathfrak{w}^{-1}(H)\right) \cdot Z & =\left[\mathfrak{w}^{-1}(H), Z\right]=\left[\mathfrak{w}^{-1}(H), \sum_{\alpha \in \Delta\left(\mathfrak{n}^{*}\right)} z_{\alpha} X_{\alpha}\right] \\
& =\sum_{\alpha \in \Delta\left(\mathfrak{n}^{*}\right)} z_{\alpha}\left[\mathfrak{w}^{-1}(H), X_{\alpha}\right] \\
& =\sum_{\alpha \in \Delta\left(\mathfrak{n}^{*}\right)} \alpha\left(\mathfrak{w}^{-1}(H)\right) z_{\alpha} X_{\alpha} \\
& =\sum_{\alpha \in \Delta\left(\mathfrak{n}^{*}\right)}(\mathfrak{w} \alpha)(H) z_{\alpha} X_{\alpha}
\end{aligned}
$$

and hence

$$
\operatorname{Exp}\left(\operatorname{ad}\left(\mathfrak{w}^{-1}(H)\right)\right) \cdot Z=\sum_{\alpha \in \Delta(\mathfrak{n})} e^{(\mathfrak{w} \alpha)(H)} z_{\alpha} X_{\alpha} .
$$

To prove $X=\bigcup_{\mathfrak{w} \in W^{1}} \mathfrak{w} \bar{N}^{*}$, we need the following fact. See [8].

FACT. If $Y$ is a compact kähler manifold and $H^{1}(Y, C)=0$, then a complex connected solvable Lie group $S$ acting holomorphically on $Y$ always has a fixed point inside any analytic subvariety that $S$ leaves invariant.

The space $X$ satisfies these assumptions and we can take $T$ as $S$. Let us apply this fact to our case. Since $\mathfrak{w} \bar{N}^{*}$ is a $T$ invariant Zariski open set, the complement $X^{\prime}=X-\bigcup_{\mathfrak{w} \in W^{1}} \mathfrak{w} \bar{N}^{*}$ becomes a $T$ invariant subvariety. Hence if $X^{\prime}$ is not empty, it must have a fixed point of $T$. But the set $W^{1}$, which is the set of all $T$ fixed points, is of course outside $X^{\prime}$. This is a contradiction.

For any $Y \in \mathfrak{g}$, we can define a holomorphic vector field $V_{Y}$ by operating $Y$ infinitesimally on $X$;

$$
\left(V_{Y} f\right)(\bar{g})=\lim _{\varepsilon \rightarrow 0} \frac{f(\exp (\varepsilon Y) \bar{g})-f(\bar{g})}{\varepsilon},
$$

where $\bar{g} \in X$ and $f$ is a local function around $\bar{g}$. In particular for an element $H \in \mathfrak{h}, V_{H}$ can be written as follows

$$
V_{H}=\sum_{\alpha \in \triangleleft\left(\mathrm{n}^{*}\right)}(\mathfrak{r} \alpha)(H) z_{\alpha} \frac{\partial}{\partial z_{\alpha}}
$$

on $\mathfrak{w} \bar{N}^{*}$ by using the local coordinate $\left\{z_{\alpha}\left(\mathfrak{w} \bar{n}^{*}\right)\right\}_{\alpha \in \Delta\left(\mathfrak{n}^{*}\right)}$. This claim is obvious from Theorem 3.3. If we choose $H$ from the Weyl chambers, we have $0 \neq(\mathfrak{w} \alpha)(H) \in \boldsymbol{R}$, for $\mathfrak{w} \in W^{1}, \alpha \in \Delta\left(\mathfrak{n}^{*}\right)$. Hence the set of all points where $V_{H}$ vanishes agrees with $W^{1}$ and $V_{H}$ vanishes with first order there. Hereafter we further assume that $H$ belongs to the positive Weyl chamber $\mathfrak{h}_{R}^{+}$. Then for $\alpha \in \Delta$, we note that $\alpha \in \Delta_{+}$if and only if $\alpha(H)>0$. 
From the expression (3.3) of $V_{H}$, if we use the local coordinate $\left\{z_{\alpha}\left(\mathfrak{w} \bar{n}^{*}\right)\right\}_{\alpha \in \Delta\left(\mathfrak{n}^{*}\right)}$ on $\mathfrak{w} \bar{N}^{*}$, any flow $P(t)$, parametrized by $t \in C$, of $V_{H}$ can be written;

$$
z_{\alpha}(P(t))=c_{\alpha}^{\mathfrak{w}} e^{(\mathfrak{w} \alpha)(H) t}, \quad c_{\alpha}^{\mathrm{w}} \in C, \quad \text { for } \alpha \in \Delta\left(\mathfrak{n}^{*}\right) .
$$

Let us introduce some notations.

$$
\begin{aligned}
\Delta_{+}\left(\mathfrak{n}^{*}, \mathfrak{w}\right) & =\left\{\alpha \in \Delta\left(\mathfrak{n}^{*}\right) \mid \mathfrak{w} \alpha \in \Delta_{+}\right\}, \\
\Delta_{-}\left(\mathfrak{n}^{*}, \mathfrak{w}\right) & =\left\{\alpha \in \Delta\left(\mathfrak{n}^{*}\right) \mid \mathfrak{w} \alpha \in \Delta_{-}\right\}, \\
\Delta\left(\mathfrak{n}^{*}, \mathfrak{w}, P(t)\right) & =\left\{\alpha \in \Delta\left(\mathfrak{n}^{*}\right) \mid c_{\alpha}^{\mathfrak{w}} \neq 0\right\}, \\
\Delta_{+}\left(\mathfrak{n}^{*}, \mathfrak{w}, P(t)\right) & =\Delta_{+}\left(\mathfrak{n}^{*}, \mathfrak{w}\right) \cap \Delta\left(\mathfrak{n}^{*}, \mathfrak{w}, P(t)\right) \\
& =\left\{\alpha \in \Delta\left(\mathfrak{n}^{*}\right) \mid c_{\alpha}^{\mathfrak{w}} \neq 0, \mathfrak{w} \alpha \in \Delta_{+}\right\}, \\
\Delta_{-}\left(\mathfrak{n}^{*}, \mathfrak{w}, P(t)\right) & =\Delta_{-}\left(\mathfrak{n}^{*}, \mathfrak{w}\right) \cap \Delta\left(\mathfrak{n}^{*}, \mathfrak{w}, P(t)\right),
\end{aligned}
$$

where $P(t)$ is the flow of $V_{H}$ on $\mathfrak{w} \bar{N}^{*}$ as above.

We divide the flow $P(t)$ of $V_{H}$ on $\mathfrak{i n} \bar{N}^{*}$ into three types;

1) $\Delta_{-}\left(\mathfrak{n}^{*}, \mathfrak{w}, P(t)\right)=\phi$, i.e., a flow starting from $\mathfrak{w}$.

2) $\Delta_{+}\left(\mathfrak{n}^{*}, \mathfrak{w}, P(t)\right)=\phi$, i.e., a flow arriving at $\mathfrak{w}$.

3) the others.

1)
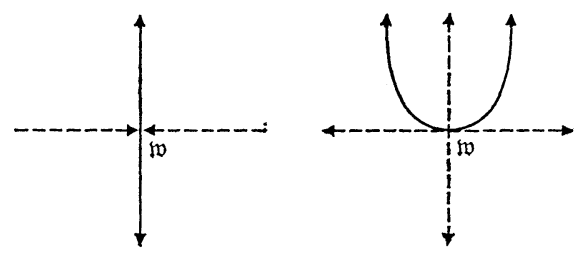

2)
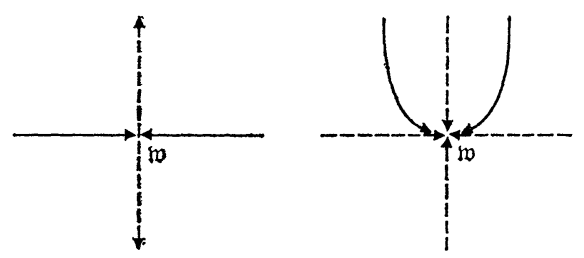

3)

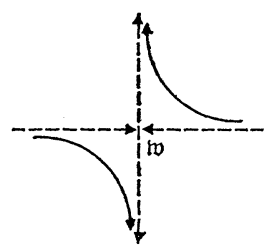

Fig. 1

For instance, let us choose a flow $P(t)$ of type 2). Then if we fix the 
imaginary part of $t$ and approach the real part of $t$ to $+\infty$, we obtain $\lim _{t \rightarrow+\infty} P(t)=\mathfrak{w}$.

\section{§4. Proof of our theorems}

Let $V_{H}$ be the same as in section 3 . Then since $\Delta\left(\mathfrak{n}^{*}\right) \subset \Delta_{-}$, we have $\alpha(H)<0$ for $\alpha \in \Delta\left(\mathfrak{n}^{*}\right)$. We further assume that $H$ satisfies

$$
\left(\mathfrak{w} \alpha_{i}\right)(H) \neq\left(\mathfrak{w} \alpha_{j}\right)(H) \quad \text { if } \alpha_{i} \neq \alpha_{j} \text { in } \Delta\left(\mathfrak{n}^{*}\right)
$$

for each $\mathfrak{w} \in W^{1}$.

We denote

$$
W^{1}(p)=\left\{\mathfrak{w} \in W^{1} \mid \text { the number of } \Delta_{+}\left(\mathfrak{n}^{*}, \mathfrak{w}\right) \text { is } p\right\}
$$

and

$$
X_{\mathfrak{w}}^{\prime}=\left\{\mathfrak{w} \bar{n}^{*} \in \mathfrak{w} \bar{N}^{*} \mid z_{\beta}\left(\mathfrak{w} \bar{n}^{*}\right)=0 \text { for } \beta \in \Delta_{-}\left(\mathfrak{n}^{*}, \mathfrak{w}\right)\right\}
$$

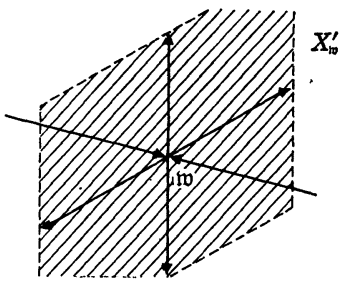

Fig. 2. $p=2$.

From the definition of $\Delta_{+}\left(\mathfrak{n}^{*}, \mathfrak{w}\right)$, the set $X_{\mathfrak{w}}^{\prime}$ can be considered as the union of all flows of $V_{H}$ which start from $\mathfrak{w}$. And if $\mathfrak{w} \in W^{1}(p), X_{\mathfrak{w}}^{\prime}$ is biholomorphic to $\boldsymbol{C}^{p}$.

Proposition 4.1. The space $X$ is decomposed into the disjoint union of the cells $X_{\mathfrak{w}}^{\prime}, \mathfrak{w} \in W^{1}$. And this decomposition is identical to the Bruhat decomposition of $X$.

Proof. We choose $x \in X$. If $x$ is fixed by the infinitesimal action of $H$, it belongs to $W^{1}$. Hence $x=\mathfrak{w} \in X_{\mathfrak{w}}^{\prime}$ for some $\mathfrak{w} \in W^{1}$. Conversely if $x$ is not fixed, there is the unique flow $P(t)$ of $V_{H}$ which passes through $x$ and the starting point $\lim _{t \rightarrow-\infty} P(t)$ is fixed by the infinitesimal action of $H$. We give the precise proof of the existence of the limit points $\lim _{t \rightarrow \pm \infty} P(t)$ in Remark 4.6 later. If we denote $\mathfrak{w}=\lim _{t \rightarrow-\infty} P(t), x$ belongs to $X_{w}^{\prime}$. To prove the equivalence of two decompositions, it suffices to show $X_{\mathfrak{w}} \supset X_{\mathfrak{w}}^{\prime}$ for each $\mathfrak{w} \in W^{1}$. We use the second description of the Bruhat 
cell, i.e., $X_{\mathfrak{w}}=M \mathfrak{w} P / P=M \mathfrak{w}$. See (2.6). Since $M$ is the maximal nilpotent Lie group contained in $B$, the Lie algebra $\mathfrak{m}$ of $M$ is written as $\mathfrak{m}=\sum_{\alpha \in \Delta_{+}} \mathfrak{g}_{\alpha}$. Then we have

$$
\begin{aligned}
X_{\mathfrak{w}} \cap \mathfrak{w} \bar{N}^{*} & =M \overline{\mathfrak{w}} \cap \mathfrak{w} \bar{N}^{*} \\
& =\mathfrak{w} \mathfrak{w}^{-1} M \mathfrak{w} \cap \mathfrak{w} \bar{N}^{*} \supset \mathfrak{w} \overline{\left(\mathfrak{w}^{-1} M \mathfrak{w}\right) \cap N^{*}} .
\end{aligned}
$$

Since the Lie algebra of $\mathfrak{w}^{-1} M \mathfrak{w} \cap N^{*}$ is

$$
\begin{aligned}
\left(\mathfrak{w}^{-1} \sum_{\beta \in \Delta_{+}} \mathfrak{g}_{\beta} \mathfrak{w}\right) \cap\left(\sum_{\alpha \in \Delta\left(\mathfrak{n}^{*}\right)} \mathfrak{g}_{\alpha}\right) & =\left(\sum_{\beta \in \Delta_{+}} \mathfrak{g}_{\mathfrak{w}-\mathfrak{1}_{\beta}}\right) \cap\left(\sum_{\alpha \in \Delta\left(\mathfrak{n}^{*}\right)} \mathfrak{g}_{\alpha}\right) \\
& =\sum_{\substack{\alpha \in \Delta\left(\mathfrak{n}^{*}\right) \\
\mathfrak{w} \alpha \in \Delta_{+}}} \mathfrak{g}_{\alpha},
\end{aligned}
$$

$\mathfrak{\mathfrak { w } ( \mathfrak { w } ^ { - 1 } M \mathfrak { w } ) \cap N ^ { * }}$ coincides with $X_{\mathfrak{w}}^{\prime}$. Hence we have $X_{\mathfrak{w}} \supset X_{\mathfrak{w}}^{\prime}$ and complete the proof.

To investigate the behavior of the flow $P(t)$ of $V_{H}$, let us embed the space $X$ into a certain projective space. See [10]. Let $\Lambda$ be an integral dominant weight such that $\left(\Lambda, \alpha_{i}\right)=0$ for $\alpha_{i} \in \Pi-\Pi(p)$ and $\left(\Lambda, \alpha_{j}\right)>0$ for $\alpha_{j} \in \Pi(\mathfrak{p})$. We denote by $(\phi, V)$ the irreducible representation of $G$ with the highest weight $\Lambda$ and by $\langle\Lambda\rangle$ the set of all weights of the representation. Here we count the weights with multiplicities. So even if the letters of weights are different as $\gamma$ and $\delta$, it does not necessarily mean that they are different as weights. The Weyl group $W$ acts on the space $\hat{\mathfrak{h}}_{\boldsymbol{R}}$ as the orthogonal transformations and the subgroup $W_{1}$ is realized as the isotropy subgroup of $W$ at $\Lambda$;

$$
W_{1}=\{\mathfrak{w} \in W \mid \mathfrak{w} \Lambda=\Lambda\} .
$$

We call such a weight as $\mathfrak{w} A, \mathfrak{w} \in W$, extremal and denote

$$
\langle\Lambda\rangle^{e}=\{\mathfrak{w} \Lambda \mid \mathfrak{w} \in W\} \subset\langle\Lambda\rangle .
$$

By virtue of the irreducibility of the representation, the multiplicity of $\mathfrak{w} A$ is one. There is a one-to-one correspondence;

$$
\begin{array}{cc}
W^{1} \longrightarrow\langle\Lambda\rangle^{e} \\
\mathcal{W} & \mathbb{W} \\
\mathfrak{W} \longrightarrow \mathfrak{w} \Lambda .
\end{array}
$$

We decompose the vector space $V$ into the weight spaces and write in the following;

$$
\boldsymbol{V}=\sum_{r \in\langle\Delta\rangle} \boldsymbol{V}_{r}
$$


From the remark stated above, $\operatorname{dim} V_{r}=1$ for all $\gamma \in\langle\Lambda\rangle$. We choose a nonzero element $Y_{r} \in V_{r}$ as the base of $V_{r}$. By virtue of the choice of $\Lambda$, the space $V_{A}$ of the highest weight is invariant by $P$ and conversely any element of $G$ which keeps $V_{A}$ invariant belongs to $P$. Hence the restriction $\left(\left.\phi\right|_{P}, V_{A}\right)$ of the representation $(\phi, V)$ is a representation of $P$ of one dimension. We write $\ell(p)=\left.\phi\right|_{P}(p), p \in P$. Then $\left.d \ell\right|_{1}=\Lambda$. The representation $\left(\ell, V_{A}\right)$ induces a homogeneous line bundle $L$ on $X=G / P$ and the space $V$ is concretely realized as the vector space $H^{\circ}(X, L)$ of all global sections of $L$. Every section $\psi \in H^{\circ}(X, L)$ can be considered as a function on $G$ satisfying

$$
\psi(g)=\ell(p) \psi(g p)
$$

for $g \in G$ and $p \in P$. The representation $\phi$ of $G$ on $V=H^{0}(X, L)$ is as follows

$$
(\phi(g) \cdot \psi)\left(g^{\prime}\right)=\psi\left(g^{-1} g^{\prime}\right)
$$

for $\psi \in H^{\circ}(X, L)$ and $g, g^{\prime} \in G$.

For simplicity, we omit the letter $\phi$ later and write in the way

$$
\phi(g) \cdot Y=g \cdot Y
$$

for $g \in G, Y \in V$. We offer the embedding theorem. See [10].

THEOREM 4.2. Let ¿ be the projection of $G$ onto the G-orbit of $Y_{A} \in V_{A}$. Since $P$ leaves the space $V_{A}$ invariant, the mapping c can be projected to $\bar{c}$;

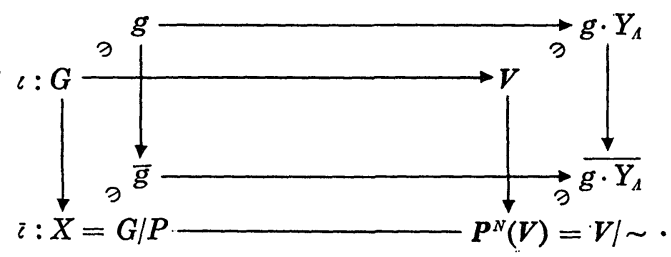

Then all the mappings above are G-equivariant. And the horizontal mapping $i$ embeds the space $X$ into $\boldsymbol{P}^{N}(V)$.

By considering the mapping

$$
\begin{gathered}
\boldsymbol{V} \longrightarrow \boldsymbol{C}^{N+1} \\
\mathbb{U} \\
\sum_{\in\langle\Lambda\rangle} x_{\gamma} Y_{r} \longrightarrow\left(x_{r}\right)_{r \in\langle\Lambda\rangle},
\end{gathered}
$$


we obtain the homogeneous coordinate $\left[x_{r}\right]_{r \in\langle\Lambda\rangle}$ of $\boldsymbol{P}^{N}(\boldsymbol{V})$. We abbreviate $\bar{Y}_{\delta}$ to $\delta$. As usual, we define affine open sets

$$
U_{\gamma}=\left\{\left[x_{\delta}\right] \in \boldsymbol{P}^{N}(V) \mid x_{\gamma} \neq 0\right\}, \quad \gamma \in\langle\Lambda\rangle,
$$

and denote by $y_{\delta}=x_{i} \mid x_{r}, \delta \in\langle\Lambda\rangle-\gamma$, the inhomogeneous coordinate on $U_{r}$.

If we denote

$$
S=\left\{\left[x_{r}\right] \in \boldsymbol{P}^{N}(V) \mid\left[x_{r}\right] \text { is fixed by the left action of } T\right\}
$$

then it is decomposed into

$$
S=\bigcup_{r \in\langle\Lambda\rangle} S_{r}, \quad \text { disjoint union },
$$

where

$S_{r}=\left\{\left[x_{\delta}\right] \in \boldsymbol{P}^{N}(V) \mid x_{\delta}=0\right.$ for any $\delta \in\langle\Lambda\rangle$ such that $\delta \neq \gamma$ as weights $\}$.

For an element $\mathfrak{w} \in N(T)$, we have

$$
\begin{aligned}
t \mathfrak{w} Y_{A} & =\mathfrak{w} \mathfrak{w}^{-1} t \mathfrak{w} Y_{A} \\
& =\mathfrak{w} \ell\left(\mathfrak{w} \mathfrak{w}^{-1} t \mathfrak{w}\right) Y_{A} \\
& =(\mathfrak{w} \ell)(t) \mathfrak{w} Y_{A}, \quad \text { for } t \in T,
\end{aligned}
$$

so $\mathfrak{w} Y_{\Lambda}$ belongs to $V_{\mathfrak{w} \Lambda}$. Hence the projected element $\mathfrak{w} \in W^{1}=N(T) / N(T)$ $\cap P \hookrightarrow X$ is mapped to $\overline{\mathfrak{w} Y_{A}}=\overline{Y_{\mathfrak{w} A}}=\mathfrak{w} \Lambda \in\langle\Lambda\rangle^{e}$ by $i$. In other words, the correspondence (4.4) coincides with $i \mid W^{1}$. The embedded manifold $i(X)$ intersects with $S$ only at the points $\langle\Lambda\rangle^{e}$. Because if $\iota(X)$ has a common point $x$ with $S$ outside $\langle\Lambda\rangle^{e}$, the inverse image $i^{-1}(x) \in X$ must be fixed by the $T$ action. But this case can not occur.

ExAmple 4.3. Let $X=G(k, n)$ be the Grassmann variety. We can write $X$ as follows

$$
X=S L(n+1, C) / P
$$

where

$$
P=\left\{A \in S L(n+1, C) \mid A=\left(\begin{array}{c|c}
A_{1} & A_{2} \\
\hline 0 & \frac{A_{3}}{2}
\end{array}\right), A_{1}:(k+1) \times(k+1) \text { matrix }\right\} .
$$

The manifold $X$ has the well known extension of vector bundles;

$$
0 \longrightarrow U \longrightarrow X \times E \longrightarrow Q \longrightarrow 0
$$

where $U$ is the universal bundle and $Q$ is the quotient bundle. The bundle $U$ is induced by the representation 


$$
\phi: P \longrightarrow G L(k+1, C)
$$

$\stackrel{\Perp}{A} \longrightarrow \quad \stackrel{\Psi}{\longrightarrow} \quad A_{1}$.

In this case, we can take $L=\operatorname{det} U^{-1}=\operatorname{det} Q$ and $V=H^{0}(X, L)$ and then we obtain the usual Plücker embedding;

$$
X \smile P^{N}(V)
$$

where $N=\left(\begin{array}{l}n+1 \\ k+1\end{array}\right)-1$. Since the Weyl group $W$ of $S L(n+1, C)$ is isomorphic to $\widetilde{S}_{n+1}$, the permutation group of $n+1$ elements, and the subgroup $W_{1}$ is isomorphic to $\mathfrak{S}_{k+1} \times \mathfrak{S}_{n-k}$, we have

$$
\text { the number of } \begin{aligned}
W^{1} & =\frac{(n+1) !}{(k+1) !(n-k) !}=\left(\begin{array}{l}
n+1 \\
k+1
\end{array}\right) \\
& =\operatorname{dim} V .
\end{aligned}
$$

Hence $\langle\Lambda\rangle^{e}=\langle\Lambda\rangle$, i.e., the weights of $(\phi, V)$ are all extremal. If we take as the basis of $V$, we obtain the usual Plücker coordinate $\left[x_{\mathrm{w}, 1}\right]_{\mathfrak{w} 1 \in\langle\Lambda\rangle}$ of $\boldsymbol{P}^{N}(\boldsymbol{V})$.

We impose one more condition on the selection of $H$;

$\delta(H) \neq \gamma(H)$ for $\gamma, \delta \in\langle\Lambda\rangle$ such that $\gamma \neq \delta$ as the elements of $\hat{\mathfrak{h}}_{R}$.

Remark 4.4. We note that the conditions imposed on the choise of $H$ are all open.

Let us lift the $T$ action on $X$ to $P^{N}(V)$ and construct a vector field $V_{H}^{\Lambda}$. Since

$$
\begin{aligned}
\lim _{\varepsilon \rightarrow 0} & \exp (\varepsilon H) \cdot\left(\sum_{r \in\langle\Lambda\rangle} x_{r} Y_{r}\right)-\left(\sum_{r \in\langle\Lambda\rangle} x_{r} Y_{r}\right) \\
\varepsilon & =\lim _{\varepsilon \rightarrow 0} \frac{\left(I+\varepsilon H / 1 !+(\varepsilon H)^{2} / 2 !+\cdots\right) \cdot\left(\sum_{r \in\langle\Lambda\rangle} x_{r} Y_{r}\right)-\left(\sum_{r \in\langle\Lambda\rangle} x_{r} Y_{r}\right)}{\varepsilon} \\
& =\sum_{r \in\langle\Lambda\rangle} \gamma(H) x_{r} Y_{r},
\end{aligned}
$$

we have a vector field $\tilde{V}_{H}^{A}$ on $V$ such that

$$
\tilde{V}_{H}^{\Lambda}=\sum_{r \in\langle\Lambda\rangle} \gamma(H) x_{r} \frac{\partial}{\partial x_{\gamma}}
$$

Because the coefficients of $\partial / \partial x_{r}, \gamma \in\langle\Lambda\rangle$, in $\tilde{V}_{H}^{\Lambda}$ are all linear functions, the vector field $\tilde{V}_{H}^{A}$ can be descended to $\boldsymbol{P}^{N}(V)$. By using the inhomoge- 
neous coordinate $\left\{y_{\delta}\right\}_{\delta \in\langle\Lambda\rangle-\gamma}$ on $U_{r}$, we can write the descended vector field $V_{H}^{A}$ as follows

$$
V_{H}^{A}=\sum_{\delta \in\langle\Lambda\rangle-\gamma}(\delta(H)-\gamma(H)) y_{\delta} \frac{\partial}{\partial y_{\delta}}=\sum_{\delta \in\langle\Lambda\rangle-r}(\delta-\gamma)(H) y_{\delta} \frac{\partial}{\partial y_{\delta}} .
$$

In general, there are multiplicities in weights so the zero set of $V_{H}^{A}$ is not necessarily isolated and in fact coincides with $S$. Every flow $P(t)$ of $V_{H}^{A}$ on $U_{r}$ can be expressed as follows

$$
y_{\delta}(P(t))=c_{\hat{\delta}} e^{(\hat{\delta}-\gamma)(H) t}, \quad c_{\hat{\delta}} \in C, \quad \text { for } \delta \in\langle\Lambda\rangle-\gamma .
$$

Proposition 4.5. 1) For any flow $P(t)$ of $V_{H}^{A}$, the limit points $\lim _{t \rightarrow \pm \infty} P(t)$ exist and belong to the zero set $S$ of $V_{H}^{A}$.

2) There is a flow of $V_{H}^{A}$ which starts from $\gamma$ and arrives at $\delta$ if and only if it is satisfied that $(\delta-\gamma)(H)>0$.

Proof. To prove 1), we can assume that a part of the flow $P(t)$ lies on $U_{r}$ for some $r \in\langle\Lambda\rangle$. Then $P(t)$ is written as (4.8) there. If the maximal value $m$ of $\left\{(\delta-\gamma)(H) \mid c_{\delta} \neq 0\right\}$ is positive, we set

$$
\begin{aligned}
M & =\left\{\delta \in\langle\Lambda\rangle \mid(\delta-\gamma)(H)=m, c_{\delta} \neq 0\right\} \\
& =\left\{\delta_{1}, \cdots, \delta_{k}\right\} .
\end{aligned}
$$

Then by using the homogeneous coordinate $\left[x_{r}\right]_{r \in\langle\Lambda\rangle}$, we have

$$
x_{\tau}\left(\lim _{t \rightarrow+\infty} P(t)\right)= \begin{cases}c_{\delta_{i}} & \text { if } \tau=\delta_{i} \in M \\ 0 & \text { if } \tau \notin M .\end{cases}
$$

From Remark 4.4., we have $\delta_{1}-\gamma=\delta_{2}-\gamma=\cdots=\delta_{k}-\gamma$ and hence $\delta_{1}$ $=\cdots=\delta_{k}$ as an element of $\hat{\mathfrak{h}}_{R}$. So the limit point $\lim _{t \rightarrow+\infty} P(t)$ exists and belongs to $S_{\delta_{1}} \subset S$. In case $m \leq 0$, the limit point $\lim _{t \rightarrow+\infty} P(t)$ also exists and belongs to $S_{r}$. Similarly we can prove the existence of $\lim _{t \rightarrow+\infty} P(t)$. If we consider the flow $P_{r}^{o}(t)$ such that

$$
y_{\tau}\left(P_{r}^{\delta}(t)\right)= \begin{cases}e^{(\delta-r)(H) t} & \text { if } \tau=\delta \\ 0 & \text { if } \tau \neq \delta\end{cases}
$$

for $\tau \in\langle\Lambda\rangle-\gamma$, we can prove 2). We omit the detailed proof.

Remark 4.6. If we embed the space $X$ into $\boldsymbol{P}^{N}(\boldsymbol{V})$, we can consider that the vector field $V_{H}$ is the restriction of $V_{H}^{A}$ to $X$. Then the flow $P(t)$ of $V_{H}$ is that of $V_{H}^{A}$ lying entirely on $X$. Hence the limit points $\lim _{t \rightarrow \pm \infty} P(t)$ always exist and belong to $\langle\Lambda\rangle^{e}$. 
In the following discussions, we consider the space $X$ as the $T$ invariant submanifold of $\boldsymbol{P}^{N}(V)$. Let us compare the expressions of $V_{H}^{A}$ and $V_{H}$ around $\mathfrak{w} \Lambda=i(\mathfrak{w})$. By using the coordinate $\left\{y_{\delta}\right\}_{\delta \in\langle\Lambda\rangle-\mathfrak{w} \Lambda}$, we have

$$
V_{H}^{A}=\sum_{\delta \in\langle\Lambda\rangle-\mathfrak{w} \Lambda}(\delta-\mathfrak{w} \Lambda)(H) y_{\delta} \frac{\partial}{\partial y_{\delta}} .
$$

On the other hand, since the space $X$ is nonsingular around $\mathfrak{w} \Lambda=\imath(\mathfrak{w})$, we add some local functions $\left\{u_{i}\right\}_{i \in I}$ to $\left\{z_{\alpha}\right\}_{\alpha \in \Delta\left(n^{*}\right)}$ and make them a local coordinate in some neighborhood $U$ of $\mathfrak{w} \Lambda=i(\mathfrak{w})$. We can assume that the functions $\left\{u_{i}\right\}_{i \in I}$ satisfy;

1) $X \cap U=\left\{x \in U \mid u_{i}(x)=0, i \in I\right\}$

2) With respect to the local coordinate $\left\{z_{\alpha}, u_{i}\right\}_{\alpha \in \Delta\left(n^{*}\right), i \in I}$, it is satisfied

$$
V_{H}^{A}=\sum_{\alpha \in \Delta\left(\mathfrak{n}^{*}\right)}\left\{(\mathfrak{w} \alpha)(H) z_{\alpha}+f_{\alpha}\left(z_{\beta} ; u_{j}\right)\right\} \frac{\partial}{\partial z_{\alpha}}+\sum_{i \in I}\left\{\gamma_{i} u_{i}+g_{i}\left(z_{\beta} ; u_{j}\right)\right\} \frac{\partial}{\partial u_{i}},
$$

where $f_{\alpha}\left(z_{\beta} ; u_{j}\right)$ and $g_{i}\left(z_{\beta} ; u_{j}\right)$ are the functions of higher terms and $f_{\alpha}\left(z_{\beta} ; 0\right)$ $=g_{i}\left(z_{\beta} ; 0\right)=0, \alpha \in \Delta\left(\mathfrak{n}^{*}\right), i \in I$. If we compare (4.9) and (4.10), we have

$$
\{(\delta-\mathfrak{w} \Lambda)(H)\}_{\delta \in\langle\Lambda\rangle-\mathfrak{w} \Lambda}=\left\{(\mathfrak{w} \alpha)(H) ; \gamma_{i}\right\}_{\alpha \in \Delta\left(\mathfrak{n}^{*}\right)}, \quad i \in I .
$$

Let us consider a flow $P_{\alpha}^{\mathfrak{w}}(t)$ with special direction of $V_{H}$ on $\mathfrak{w} \bar{N}^{*}$ such that

$$
z_{\beta}\left(P_{\alpha}^{\mathfrak{w}}(t)\right)= \begin{cases}c_{\alpha}^{\mathfrak{t}} e^{(\mathfrak{w} \alpha)(H) t} & \text { if } \beta=\alpha \\ 0 & \text { if } \beta \neq \alpha,\end{cases}
$$

where $C \ni c_{\alpha}^{\mathfrak{w}} \neq 0$. In other words, $\Delta\left(\mathfrak{n}^{*}, \mathfrak{w}, P_{\alpha}^{\mathfrak{w}}(t)\right)=\alpha$. If $\mathfrak{w} \alpha \in \Delta_{+}$then $P_{\alpha}^{\mathfrak{w}}(t)$ starts from $\mathfrak{w}$. Conversely if $\mathfrak{w} \alpha \in \Delta_{-}, P_{\alpha}^{\mathfrak{w}}(t)$ arrives at $\mathfrak{w}$.

Proposition 4.7. Let $P_{\alpha}^{\mathfrak{w}}(t)$ be the flow of $V_{H}$ on $\mathfrak{w} \bar{N}^{*}$ as above. We assume $\mathfrak{w} \alpha \in \Delta_{+}$and put $\mathfrak{w}^{\prime}=\lim _{t \rightarrow+\infty} P_{\alpha}^{\mathfrak{w}}(t) \in W^{1}$. If we write the flow by using the local coordinate $\left\{z_{\beta}\left(\mathfrak{w}^{\prime} \bar{n}^{*}\right)\right\}_{\beta \in \Delta\left(\mathfrak{n}^{*}\right)}$ around $\mathfrak{w}^{\prime}$, it follows

$$
z_{\beta}\left(P_{\alpha}^{\mathfrak{w}}(t)\right)= \begin{cases}c_{\alpha}^{\mathfrak{W} \prime} e^{\left(\mathfrak{w}^{\prime} \beta\right)(H) t} & \text { if } \mathfrak{w} \alpha=-\mathfrak{w}^{\prime} \beta \\ 0 & \text { otherwise } .\end{cases}
$$

Namely the flows $P_{\alpha}^{\mathfrak{w}}(t)$ and $P_{\beta}^{\mathfrak{w}}(t)$ agree with each other on $\mathfrak{w} \bar{N}^{*} \cap \mathfrak{w}^{\prime} \bar{N}^{*}$.

Proof. To avoid confusion, we write the local coordinate $\left\{z_{\beta}\left(\mathfrak{m} \mathfrak{n} \bar{n}^{*}\right)\right\}_{\beta \in \Delta\left(\mathfrak{n}^{*}\right)}$ as $\left\{z_{\beta}\right\}_{\beta \in \Delta\left(\mathfrak{n}^{*}\right)}$ and the local coordinate $\left\{z_{\beta}\left(\mathfrak{w}^{\prime} \bar{n}^{*}\right)\right\}_{\beta \in \Delta\left(\mathfrak{n}^{*}\right)}$ as $\left\{z_{\beta}^{\prime}\right\}_{\beta \in \Delta\left(\mathfrak{n}^{*}\right)}$. We choose a point $P_{\alpha}^{\mathrm{w}}\left(t_{0}\right), 0 \neq t_{0} \in C$, on the flow and put $z_{\beta}\left(P_{\alpha}^{\mathrm{w}}\left(t_{0}\right)\right)=z_{\beta, 0}, \beta \in$ $\Delta\left(\mathfrak{n}^{*}\right)$, and $z_{\beta}^{\prime}\left(P_{\alpha}^{\mathfrak{w}}\left(t_{0}\right)\right)=z_{\beta, 0}^{\prime}, \beta \in \Delta\left(\mathfrak{n}^{*}\right)$. Then both $\left\{z_{\beta}-z_{\beta, 0}\right\}_{\beta \in \Delta\left(\mathfrak{n}^{*}\right)}$ and $\left\{z_{\beta}^{\prime}-\right.$ 
$\left.\boldsymbol{z}_{\beta, 0}^{\prime}\right\}_{\beta \in \Delta\left(\mathrm{n}^{*}\right)}$ are local coordinates around $P_{\alpha}^{\mathfrak{w}}\left(t_{0}\right)$. If we compare the expressions of the flow $P_{\alpha}^{\mathrm{w}}(t)$ by using the two local coordinate, we have

$$
\begin{aligned}
z_{\beta}^{\prime}\left(P_{\alpha}^{\mathfrak{w}}(t)\right)-z_{\beta, 0}^{\prime} & =\sum_{n=0}^{\infty} d_{\beta, n}\left(z_{\alpha}\left(P_{\alpha}^{\mathfrak{w}}(t)\right)-z_{\alpha, 0}\right)^{n} \\
& =\sum_{n=0}^{\infty} d_{\beta, n}\left(c_{\alpha}^{\mathfrak{w} w} e^{(\mathfrak{w} \alpha)(H) t}-z_{\alpha, 0}\right)^{n} \\
& =\sum_{n=0}^{\infty} d_{\beta, n}^{\prime} e^{n(\mathfrak{w} \alpha)(H) t}
\end{aligned}
$$

and hence

$$
z_{\beta}^{\prime}\left(P_{\alpha}^{\mathfrak{w}}(t)\right)=\sum_{n=0}^{\infty} d_{\beta, n}^{\prime \prime} e^{n(w \alpha)(H) t} \quad \text { for } \beta \in \Delta\left(\mathfrak{n}^{*}\right) .
$$

On the other hand, any flow $P(t)$ of $V_{H}$ on $\mathfrak{w}^{\prime} \bar{N}^{*}$ must be written as follows

$$
z_{\beta}^{\prime}(P(t))=c_{\beta}^{\mathfrak{w}^{\prime}} e^{\left(\mathfrak{i} \mathfrak{w}^{\prime} \beta\right)(H) t}, \quad c_{\beta}^{\mathfrak{w} \mathrm{w}^{\prime}} \in C, \quad \text { for } \beta \in \Delta\left(\mathfrak{n}^{*}\right) .
$$

Hence if we compare the coefficients of $t$ in (4.12) and (4.13), we have

$$
c_{\beta}^{\mathfrak{w}^{\prime}}\left(\mathfrak{w}^{\prime} \beta\right)(H)=\left(\sum_{n=0}^{\infty} n d_{\beta, n}^{\prime \prime}\right)(\mathfrak{w} \alpha)(H) .
$$

From Remark 4.4., this means

$$
c_{\beta}^{\mathfrak{w} \mathfrak{w}^{\prime}}\left(\mathfrak{w}^{\prime} \beta\right)=\left(\sum_{n=0}^{\infty} n d_{\beta, n}^{\prime \prime}\right)(\mathfrak{w} \alpha)
$$

as an element of $\hat{\mathfrak{h}}_{R}$. We note that if $\alpha \in \Delta$ and $c \alpha \in \Delta$ then $c= \pm 1$. So if $c_{\beta}^{\mathfrak{w}^{\prime}} \neq 0$ then $\left(\sum_{n=0}^{\infty} n d_{\beta, n}^{\prime \prime}\right) / c_{\beta}^{\mathfrak{w}^{\prime}}= \pm 1$ and $\mathfrak{w}^{\prime} \beta= \pm \mathfrak{w} \alpha$. Further since the flow $P_{\alpha}^{\mathfrak{w}}(t)$ arrives at $\mathfrak{w}^{\prime}$, we have $\mathfrak{w}^{\prime} \beta=-\mathfrak{w} \alpha$ and $\beta=-\mathfrak{w}^{\prime-1} \mathfrak{w} \alpha$. We complete the proof.

Remark 4.8. From the above proposition, it is easily shown that the flow $P_{\alpha}^{\mathfrak{w}}(t)$ does not lie on any open set $\mathfrak{w}^{\prime \prime} \bar{N}^{*}, \mathfrak{w}^{\prime \prime} \in W^{1}$, except $\mathfrak{i} \bar{N}^{*}$ and $\mathfrak{w}^{\prime} \overline{N^{*}}$.

Theorem 4.9. Let $P_{\alpha}^{\mathfrak{w}}(t)$ be the flow as (4.11) and assume $\mathfrak{w} \alpha \in \Delta_{+}$, i.e., $\lim _{t \rightarrow-\infty} P_{\alpha}^{\mathfrak{w}}(t)=\mathfrak{w}$. We consider the space $X$ as the $T$ invariant submanifold of $\boldsymbol{P}^{N}(\boldsymbol{V})$ and identify the point $\mathfrak{w} \in W^{1}$ and $\mathfrak{w} \Lambda \in\langle\Lambda\rangle^{e}$. Then the following three conditions are all equivalent;

1) $\lim _{t \rightarrow+\infty} P_{\alpha}^{\mathfrak{w}}(t)=\mathfrak{w}^{\prime} \Lambda \in\langle\Lambda\rangle^{e}$, where

2) $\mathfrak{w}^{\prime} \Lambda-\mathfrak{w} \Lambda=-g \cdot \mathfrak{w} \alpha \in \hat{\mathfrak{h}}_{R}$, 


$$
g=\frac{2(\Lambda, \alpha)}{(\alpha, \alpha)}
$$

3) $\mathfrak{w}^{-1} S_{\mathfrak{w} \alpha} \mathfrak{w}^{\prime} \in W_{1}$.

Proof. If we change the local coordinate from $\left\{z_{\alpha} ; u_{i}\right\}_{\alpha \in \Delta\left(\mathrm{n}^{*}\right), i \in I}$ to $\left\{y_{\delta}\right\}_{\delta \in\langle\Lambda\rangle-\mathfrak{w} \Lambda}$ around $i(\mathfrak{W})=\mathfrak{w} \Lambda$ then we have the convergence power series

$$
\begin{aligned}
y_{\delta}\left(P_{\alpha}^{\mathfrak{w}}(t)\right) & =\sum_{n=0}^{\infty} d_{\delta, n}\left(c_{\alpha}^{\mathfrak{w}} e^{(\mathfrak{w} \alpha)(H) t}\right)^{n} \\
& =\sum_{n=0}^{\infty} d_{\delta, n}^{\prime} e^{n(\mathrm{w} \alpha)(H) t}
\end{aligned}
$$

for $\delta \in\langle\Lambda\rangle-\mathfrak{w} \Lambda$. But any flow $P(t)$ of $V_{H}^{\Lambda}$ on $U_{\mathrm{w} \Lambda}$ must be written as (4.8). So if we compare the coefficients of $t$ in (4.8) and (4.14), we have

$$
b_{\delta}(\delta-\mathfrak{w} \Lambda)(H)=\left(\sum_{n=0}^{\infty} n d_{\delta, n}^{\prime}\right)(\mathfrak{w} \alpha)(H) .
$$

And from Remark 4.4, we have

$$
b_{\delta}(\delta-\mathfrak{w} \Lambda)=\left(\sum_{n=0}^{\infty} n d_{\delta, n}^{\prime}\right)(\mathfrak{w} \alpha)
$$

as an element of $\hat{\mathfrak{h}}_{R}$.

The limit point $\lim _{t \rightarrow+\infty} P_{\alpha}^{\mathfrak{w}}(t)$ is determined by comparing the magnitude of the values $\left\{(\delta-\mathfrak{w} \Lambda)(H) \mid b_{\delta} \neq 0\right\}$. And from Remark 4.6, there must exist at least one element $\mathfrak{w}^{\prime} \Lambda \in\langle\Lambda\rangle^{e}-\mathfrak{w} \Lambda$ such that $b_{\mathfrak{w}^{\prime} \Lambda} \neq 0$. Then we have

$$
b_{\mathfrak{w} \prime} \Lambda\left(\mathfrak{W} \mathfrak{W}^{\prime} \Lambda-\mathfrak{w} \Lambda\right)=\left(\sum_{n=0}^{\infty} n d_{\mathfrak{w} \Lambda, n}^{\prime}\right)(\mathfrak{w} \alpha) \in \hat{\mathfrak{h}}_{\boldsymbol{R}}
$$

and hence

$$
\mathfrak{w}^{\prime} \Lambda-\mathfrak{w} \Lambda=\mathfrak{c} \mathfrak{w} \alpha,
$$

where

$$
c=\left(\sum_{n=0}^{\infty} n d_{\mathfrak{r} \Lambda, n}^{\prime}\right) / b_{\mathfrak{w}^{\prime} \Lambda} .
$$

Fortunately this condition determines the value $c$ uniquely as

$$
c=-g=-2 \frac{(\Lambda, \alpha)}{(\alpha, \alpha)}
$$

and consequently the extremal weight $\mathfrak{w}^{\prime} \Lambda$ is also uniquely determined. If 


$$
c \neq-g=-2 \frac{(\Lambda, \alpha)}{(\alpha, \alpha)},
$$

we have three distinct extremal weights $\Lambda, \mathfrak{w}^{-1} \mathfrak{w}^{\prime} \Lambda=\Lambda+c \alpha$ and $S_{\alpha} \Lambda=\Lambda$ - g $\alpha$ lying on a straight line. But since the elements of the Weyl group $W$ act on $\hat{\mathfrak{h}}_{\boldsymbol{R}}$ as orthogonal transformations with respect to the norm (, ) determined by the Killing form, the points $\Lambda, \mathfrak{w}^{-1} \mathfrak{w}^{\prime} \Lambda$ and $S_{\alpha} \Lambda$ also lie on the hypersphere of radius $|\Lambda|=(\Lambda, \Lambda)^{1 / 2}$. This is a contradiction. Hence we have

$$
c=-g=-2 \frac{(\Lambda, \alpha)}{(\alpha, \alpha)}
$$

and then $\mathfrak{w}^{-1} \mathfrak{w}^{\prime} \Lambda=\Lambda-g \alpha=S_{\alpha} \Lambda$. Since $W_{1}$ is the isotropy subgroup of $W$ at $\Lambda$, we have $S_{\alpha} \mathfrak{\mathfrak { W } ^ { - 1 }} \mathfrak{\mathfrak { W } ^ { \prime }}=\mathfrak{w}^{-1} \mathfrak{w} S_{\alpha} \mathfrak{\mathfrak { w } ^ { - 1 }} \mathfrak{w}^{\prime}=\mathfrak{w}^{-1} S_{\mathfrak{w} \alpha} \mathfrak{\mathfrak { W } ^ { \prime }} \in W_{1}$. We complete the proof.

Remark 4.10. In the case $X=G / B$, we have $W_{1}=\{1\}$. So the condition 3) means $S_{\mathfrak{w a}} \mathfrak{w}^{\prime}=\mathfrak{w}$.

Remark 4.11. If we change the representatives $\mathfrak{w}, \mathfrak{w}^{\prime}$ of $\overline{\mathfrak{w}}, \overline{\mathfrak{w}}^{\prime} \in W^{1}=$ $W / W_{1}$ to $\mathfrak{w}_{1}, \mathfrak{w}^{\prime} \mathfrak{w}_{1}^{\prime}$ respectively, where $\mathfrak{w}_{1}, \mathfrak{w}_{1}^{\prime} \in W_{1}$, we have

$$
\mathfrak{w}^{-1} S_{\mathfrak{w} \alpha} \mathfrak{W}^{\prime}=\mathfrak{w}_{1}\left(\mathfrak{W} \mathfrak{w}_{1}\right)^{-1} S_{\left(\mathfrak{w w}_{1}\right)\left(\mathfrak{w}_{1}^{-1} \alpha\right)}\left(\mathfrak{w}^{\prime} \mathfrak{w}_{1}^{\prime}\right) \mathfrak{w}_{1}^{\prime-1} \in W_{1}
$$

and hence

$$
\left(\mathfrak{w} \mathfrak{W}_{1}\right)^{-1} S_{\left(\mathfrak{w w w}_{1}\right)\left(\mathfrak{w}_{1}^{-1} \alpha\right)}\left(\mathfrak{w}^{\prime} \mathfrak{w}_{1}^{\prime}\right) \in W_{1} .
$$

We note that from Remark 2.1., $\mathfrak{w}_{1}^{-1} \alpha$ belongs to $\Delta\left(\mathfrak{n}^{*}\right)$.

THEOREM 4.12. For an element $\mathfrak{w}^{\prime} \in W^{1}$, the following are equivalent:

1) $\bar{X}_{\mathfrak{w}^{\prime}}$ is contained in $\bar{X}_{\mathfrak{w}}$, i.e., $\mathfrak{w}^{\prime} \leq \mathfrak{w}$,

2) $\mathfrak{w}^{\prime}$ belongs to $\bar{X}_{\mathfrak{w}}$,

3) There exist two series $\mathfrak{w}=\mathfrak{w}_{0}, \mathfrak{w}_{1}, \cdots, \mathfrak{w}_{k}=\mathfrak{w}^{\prime} \in W^{1}$ and $\alpha_{0}, \alpha_{1}, \cdots$ $\alpha_{k-1} \in \Delta\left(\mathfrak{n}^{*}\right)$ such that

$$
\mathfrak{w}_{i}^{-1} S_{\mathfrak{w}_{i} \alpha_{i}} \mathfrak{w}_{i+1} \in W_{1} \quad \text { and } \quad \mathfrak{w}_{i} \alpha_{i} \in \Delta_{+}, \quad 0 \leq i \leq k-1 .
$$

Proof. 1) $\Rightarrow 2$ ) is obvious. 2) $\Rightarrow 1$ ). If $\mathfrak{w}^{\prime}$ belongs to $\bar{X}_{\mathfrak{w}}$, since $\bar{X}_{\mathfrak{w}}$ is $N$ invariant, $X_{w^{\prime}}=N \overline{\mathfrak{w}}^{\prime} \subset \bar{X}_{\mathfrak{w}}$. Hence we have $\bar{X}_{\mathfrak{w}^{\prime}} \subset \bar{X}_{\mathfrak{w}}$. 3) $\Rightarrow 2$ ). By virtue of Theorem 4.9., we have

$$
\mathfrak{w}_{1}=\lim _{t \rightarrow+\infty} P_{\alpha_{0}}^{\mathfrak{w}_{0}}(t) \in \bar{X}_{\mathfrak{w}_{0}} \quad \text { and } \quad \mathfrak{w}_{2}=\lim _{t \rightarrow+\infty} P_{\alpha_{1}}^{\mathfrak{w}_{1}}(t) \in \bar{X}_{\mathfrak{w}} \subset \bar{X}_{\mathfrak{w}_{0}}
$$


Repeating this procedure we can show $\mathfrak{w}_{k}=\mathfrak{w}^{\prime} \in \bar{X}_{\mathfrak{w}}$. Let us prove the converse 2) $\Rightarrow 3$ ) by induction on the dimension of $\bar{X}_{w}$. If $\operatorname{dim} \bar{X}_{w}=0$, it is obvious. Hence we assume that if $\operatorname{dim} \bar{X}_{\mathfrak{w}}=n-1$, the assertion has been already proved. Put $\operatorname{dim} \bar{X}_{\mathfrak{w}}=n$ and choose an element $\mathfrak{w}^{\prime} \in W^{1}$ which belongs to $\bar{X}_{w}$. If $\operatorname{dim} \bar{X}_{w^{\prime}} ફ n-1$, we select $\bar{X}_{w^{\prime \prime}}$ such that $\bar{X}_{w^{\prime}} \subset \bar{X}_{w^{\prime \prime}} \subset$ $\bar{X}_{\mathfrak{w}}$ and $\operatorname{dim} \bar{X}_{\mathfrak{w}}=n-1$. From the induction there exist two series $\mathfrak{w}^{\prime \prime}$ $=\mathfrak{w}_{0}, \mathfrak{w}_{1}, \cdots, \mathfrak{w}_{k-1}=\mathfrak{w}^{\prime} \in W^{1}$ and $\alpha_{0}, \alpha_{1}, \cdots, \alpha_{k-2} \in \Delta\left(\mathfrak{n}^{*}\right)$ such that

$$
\mathfrak{w}_{i}^{-1} S_{\mathfrak{w}_{i} \alpha_{i}} \mathfrak{w}_{i+1} \in W_{1} \quad \text { and } \quad \mathfrak{w}_{i} \alpha_{i} \in \Delta_{+}, \quad 0 \leq i \leq k-2 \text {. }
$$

So it is sufficient to show that if $\operatorname{dim} \bar{X}_{\mathfrak{w}^{\prime}}=n-1$ and $\bar{X}_{\mathfrak{w}^{\prime}} \subset \bar{X}_{\mathfrak{w}}$, there exists an element $\alpha \in \Delta\left(\mathfrak{n}^{*}\right)$ such that

$$
\mathfrak{w}^{-1} S_{\mathfrak{w} \alpha} \mathfrak{w}^{\prime} \in W_{1} \text { and } \mathfrak{w} \alpha \in \Delta_{+} .
$$

We denote

$$
U_{\mathfrak{w}^{\prime}}=\left\{x \in \mathfrak{w}^{\prime} \bar{N}^{*} \mid z_{\alpha}(x)=0 \text { for any } \alpha \in \Delta_{+}\left(\mathfrak{n}^{*}, \mathfrak{w}^{\prime}\right)\right\} .
$$

Namely, $U_{\mathfrak{w}^{\prime}}$ is the union of all flows of $V_{H}$ which arrives at $\mathfrak{w}^{\prime}$. Then $U_{\mathfrak{w}^{\prime}}$ is invariant by the action of $T$ and $\operatorname{dim} U_{\mathfrak{w}^{\prime}}=\operatorname{dim} X-\operatorname{dim} \bar{X}_{\mathfrak{w}^{\prime}}=$ $\operatorname{dim} \mathfrak{n}^{*}-(n-1)$. From the definition of $U_{\mathfrak{w}^{\prime}}$, we have $\mathfrak{w}^{\prime}=X_{\mathfrak{w}^{\prime}} \cap U_{\mathfrak{w}^{\prime}} \subseteq$ $\bar{X}_{\mathfrak{w}} \cap U_{\mathfrak{w}^{\prime}}$ and $\operatorname{dim} \bar{X}_{\mathfrak{w}} \cap U_{\mathfrak{w}^{\prime}}=1$. So there exists an element $x$ such that $\mathfrak{w}^{\prime} \neq x \in \bar{X}_{\mathfrak{w}} \cap U_{\mathfrak{w}^{\prime}}$. By virtue of $T$ invariantness of the both sets $\bar{X}_{\mathfrak{w}}$ and $U_{\mathfrak{w}^{\prime}}$, the flow $P(t)$ of $V_{H}$ which passes through $x$ lies entirely on $\bar{X}_{\mathfrak{w}} \cap U_{\mathfrak{w}}$. And from the definition of $U_{\mathfrak{w}^{\prime}}, \lim _{t \rightarrow+\infty} P(t)=\mathfrak{w}^{\prime}$. If $\lim _{t \rightarrow-\infty} P(t)=\mathfrak{w}^{\prime \prime} \neq \mathfrak{w}$ then we have $\bar{X}_{w^{\prime}} \subsetneq \bar{X}_{w^{\prime \prime}} \subsetneq \bar{X}_{w}$. This contradicts to our assumption. So $\lim _{t \rightarrow-\infty} P(t)=\mathfrak{w}$. The flow $P(t)$ starts from in so $\Delta_{-}\left(\mathfrak{n}^{*}, \mathfrak{w}, P(t)\right)=\phi$. We denote

$$
T \cdot P=\left\{\exp \left(H^{\prime}\right) \cdot P(t) \in X \mid H^{\prime} \in \mathfrak{h}, t \in C\right\} .
$$

From the $T$ invariantness of $\bar{X}_{w}$ and $U_{\mathfrak{w}}$, the set $T \cdot P$ is contained in $\bar{X}_{\mathfrak{w}}$ $\cap U_{\mathfrak{w}^{\prime}}$. If the number of $\Delta_{+}\left(\mathfrak{n}^{*}, \mathfrak{w}, P(t)\right)$ is greater than two, from Theorem 3.3., $\operatorname{dim} T \cdot P \geqq 2$. This is a contradiction. Hence there exists an element $\alpha \in \Delta\left(\mathfrak{n}^{*}\right)$ such that $\alpha=\Delta_{+}\left(\mathfrak{n}^{*}, \mathfrak{w}, P(t)\right)$ and then the flow $P(t)$ coincides with the distinguished flow $P_{\alpha}^{\mathrm{r}}(t)$. By virtue of Theorem 4.9., we complete the proof.

Remark 4.13. From Remark 4.11 the condition 3) is independent of the choice of the representatives of $W^{1}$ in $W$.

Example 4.14. Let $\boldsymbol{P}^{3}=S L(4, C) / P$, where 


$$
P=\left\{\left(\begin{array}{c|c}
*_{1} & *_{2} \\
\hline 0 & \\
0 & *_{3} \\
0 &
\end{array}\right\} \in S L(4, C)\right\} .
$$

The Weyl group $W$ of $S L(4, C)$ is isomorphic to $\widetilde{\varsigma}_{4}$ and the subgroup $W_{1}$ is isomorphic to $\{1\} \times \mathfrak{S}_{3}$. Hence we have $W^{1}=\left\{\mathfrak{w}_{0}, \mathfrak{w}_{1}, \mathfrak{w}_{2}, \mathfrak{w}_{3}\right\}$. We can assume $\mathfrak{w}_{i} \in W^{1}(i), 0 \leq i \leq 3$. We obtain the following figure.

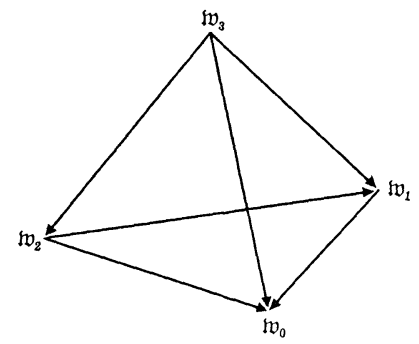

Fig. 3

\section{REFERENCES}

[1] I. N. Bernstein, I. M. Gel'fand and S. I. Gel'fand, Schubert cells and cohomology of the spaces $G / P$, Russ. Math. Surveys, 28 (1975), 1-26.

[2] - Differential operators on the base affine space and a study of g-modules, Lie groups and their representations, Halsted Press (1975), 21-64.

[ 3 ] J. Dixmier, Enveloping algebras, North-Holland Mathematical Library, Vol. 14.

[4] V. V. Deodhar, Some characterizations of Bruhat ordering on a Coxter group and determination of the relative Möbius function, Inventiones Math., 39 (1977), 187198.

[5] Y. Kato, On the vector fields on an algebraic homogeneous space, preprint.

[6] B. Kostant, Lie algebra cohomology and generalized Borel-Weil theorem, Ann. Math., 74(2) (1961), 329-387.

[7] - Lie algebra cohomology and generalized Schubert cells, Ann. Math., 77(1) (1963), 72-144.

[ 8 ] A. J. Sommese, Holomorphic vector fields on compact kaehler manifolds, Math. Ann., 210 (1974), 75-82.

[ 9 ] V. S. Varadarajan, Lie groups, Lie algebras and their representations, PrenticeHall, Inc.

[10] N. Wallach, Harmonic analysis on homogeneous spaces, Marcel Dekker, Inc.

[11] G. Warner, Harmonic analysis on semisimple Lie groups I, Springer Verlag.

Nagoya University 